\title{
LEVANTAMENTO E ESPACIALIZAÇÃO DA CRIMINALIDADE URBANA DO MUNICÍPIO DE MOSSORÓ-RN
}

C. S. P. SILVA* A. M. GRIGIO e M. R. C. PIMENTA
Universidade do Estado do Rio Grande do Norte - UERN
camilasaiury@hotmail.com*
Artigo submetido em fevereiro/2016 e aceito em março/2016
DOI: $10.15628 /$ holos. 2016.4187

\section{RESUMO}

O aumento da criminalidade, sobretudo em áreas urbanas tem provocado um sentimento de medo e insegurança pública, que ao longo do tempo vêm contribuindo para deterioração da qualidade de vida do homem nas cidades. Nesse contexto, o mapeamento da criminalidade surge como uma importante ferramenta que possibilita a identificação de áreas vulneráveis, dando suporte ao desenvolvimento de ações preventivas ao aumento da criminalidade. Este artigo teve como objetivo a distribuição espaço-temporal dos casos de homicídios ocorridos na zona urbana do município de
Mossoró entre os anos de 2010 a 2013. Utilizou-se de dados secundários e quantitativos do Instituto TécnicoCientífico de Polícia e Sistema de Informações Geográficas para a espacialização. A partir do mapeamento foi possível concluir que os bairros com maiores incidências foram o Santo Antônio, Barrocas, Aeroporto, Santa Delmira e Dom Jaime Câmara, estes apresentando condições socioeconômicas e ambientais precárias, pouco assistidos pela infraestrutura urbana e equipamentos comunitários.

PALAVRAS-CHAVE: Planejamento Urbano, Segurança Pública, Criminalidade Urbana.

\section{SURVEY AND SPATIALIZATION CRIME URBAN COUNTY MOSSORÓ-RN}

\section{ABSTRACT}

The increase in crime, especially in urban areas has caused a feeling of fear and public insecurity, which over time have been contributing to the deterioration of the quality of life of man in the cities. In this context, the mapping of crime emerges as an important tool that enables the identification of vulnerable areas, giving support to the development of preventive actions to increase in crime. This article had as objective the spatialtemporal distribution of the cases of homicides in the urban area of the city of Mossoró between the years 2010
\end{abstract}

to 2013. We used secondary data and quantitative limits of the Institute's Technical-Scientific of Police and Geographic Information System for the spatialisation. From mapping was possible to conclude that the districts with higher incidences were the Santo Antônio, Barrocas, Aeroporto, Santa Delmira and Dom Jaime Câmara, these presenting precarious socioeconomic and environmental conditions, little assisted by urban infrastructure and community equipment.

KEYWORDS: Urban Planning, Public Safety, Urban Crime. 


\section{INTRODUÇÃO}

Nas últimas décadas o Brasil vem passando por um acelerado processo de urbanização, atualmente mais de $80 \%$ de sua população reside em áreas urbanas (IBGE, 2011), o que vem gerando preocupações em relação à qualidade de vida do homem nas cidades, haja vista que o crescimento urbano, sem o devido planejamento, tem gerado uma série de consequências negativas por meio da falta de acesso de parte da população aos serviços públicos essenciais, como moradia, saúde, educação, lazer, emprego e segurança, contribuindo para um cenário de vulnerabilidade socioambiental.

Nesse contexto de vulnerabilidade, uma das consequências tem sido o aumento significativo nos índices de criminalidade. Alguns estudos apontam que o aumento das taxas de criminalidade nas cidades está associado ao acelerado crescimento urbano, a desordem, a degradação física do ambiente, a falta de acesso a equipamentos comunitários e a falta de infraestrutura urbana, fatores esses, que exercem influência direta sobre a ocorrência dos crimes (BEATO, 2008).

Os meios de comunicação de massa exibem o expressivo aumento da violência e da criminalidade urbana, apresentando os elevados números de homicídios ocorridos nas cidades, provocando um sentimento de medo e insegurança pública que ao longo do tempo vêm contribuindo para deterioração da qualidade de vida do homem nas cidades.

Nos dias atuais a capacidade de gerar informações como subsídio para a tomada de decisões é uma das questões mais importantes para o desenvolvimento estratégico. Desta forma, a carência de pesquisas sobre criminalidade tem se constituído um obstáculo para a elaboração de políticas públicas. Assim, o mapeamento dos municípios surge como uma importante ferramenta, que possibilita a identificação de áreas problemáticas, dando suporte ao planejamento de ações preventivas ao aumento da criminalidade urbana.

Mossoró é considerada uma das cidades de médio porte no contexto brasileiro e depois da capital, Natal, a maior aglomeração urbana e populacional do estado do Rio Grande do Norte. Nas últimas décadas vem passando por um intenso crescimento urbano que afetou o modelo espacial da cidade, assim como as relações sociais nela estabelecidas (SALLES, 2013).

Dado este contexto, o presente artigo buscou espacializar a criminalidade urbana, a partir dos casos de homicídios ocorridos entre 2010 a 2013 para identificar os bairros de maior incidência na zona urbana do município de Mossoró. Para o mapeamento utilizou-se dados secundários e quantitativos do Instituto Técnico-Científico de Polícia, Sistema de Informações Geográficas software ArcGIS 10.1 e base cartográfica do Instituto Brasileiro de Geografia e Estatística.

\section{PROCESSO DE URBANIZAÇÃO E CRIMINALIDADE}

O Brasil é um país urbanizado, uma média de $84 \%$ de sua população, do total de 190 milhões de pessoas, vive em cidades, onde a taxa de crescimento populacional é de 1,17\% ao ano. Em cinquenta anos, a população urbana no Brasil aumentou cinco vezes. O processo de crescimento urbano no Brasil se deu de forma rápida e desordenada. A população urbana brasileira cresceu vertiginosamente, enquanto a população rural diminuiu significativamente. Em 1960 a população 
urbana representava $45 \%$ da população total. Nos anos de 1970, mais da metade da população brasileira já residia em áreas urbanas, representando 56\%. No início da década de 1990 a população urbana já alcançava os $75 \%$ e nos anos 2000 , 81\% (IBGE, 2011).

Para Menegat e Almeida (2004) o crescimento urbano brasileiro ocorreu de forma desequilibrada em diversos sentidos. Por um lado, a distribuição territorial da malha urbana era bastante heterogênea, com a maior parte da população vivendo em grandes cidades. Por outro, a desigualdade social nas cidades também aumentou. Os autores ressaltam que os setores populacionais mais pobres foram aqueles que mais se multiplicaram em relação aos ricos.

Cerca de $60 \%$ da população brasileira vive com até um salário mínimo. Situação essa que muitas vezes obriga algumas populações a ocupar áreas periféricas da cidade onde demandam menos recursos financeiros para aquisição de imóveis, tendo em vista a desvalorização imobiliária, falta de infraestrutura urbana e acesso aos equipamentos comunitários da cidade (IBGE, 2010).

Para Silva e Travassos (2008) as elevadas taxas de crescimento e adensamento populacional aliado à concentração da pobreza nos grandes centros urbanos exercem interferência direta na configuração espacial das cidades. As autoras expõem que uma parcela significativa da população de baixa renda encontra o seu local de moradia nas periferias urbanas.

A cidade informal se proliferou com taxas muito maiores do que em relação à cidade formal:

Grandes contingentes populacionais vivem em situação de total inexistência da estrutura urbana básica: habitação, água potável, saneamento e transporte. Além disso, a estrutura político administrativa dos municípios não acompanhou o surgimento de novas demandas derivadas das elevadas taxas de urbanização que se verificaram no período, mesmo porque os governos locais sempre tiveram pouca importância na estrutura da administração pública brasileira (MENEGAT; ALMEIDA, 2004, p. 175).

Essa migração do campo para a cidade não foi acompanhada pelo planejamento e pela estruturação das cidades, o que contribuiu para a degradação socioambiental:

Além da falta de planejamento por parte do Poder Público, verificada no processo de urbanização, a falta de infra-estrutura dos serviços públicos essenciais contribuiu para a realidade de segregação e desigualdade social vivenciada nas grandes cidades. Diversos são os problemas urbanos: a falta de saneamento básico, a poluição atmosférica, a destinação indevida aos resíduos sólidos, a violência e o trânsito. Todos contribuem com a degradação do meio ambiente urbano que, por sua vez, gera a degradação da qualidade de vida da população (PUGLISI, 2006, p.101).

Assim todas as cidades brasileiras exibem problemas de habitação, transportes, lazer, água, esgotos, educação e saúde de forma semelhante, sendo diferenciado apenas o grau e a intensidade de ocorrência dos mesmos. Santos $(2009$, p. 105) destaca que "quanto maior a cidade, mais visíveis se tornam essas mazelas".

De acordo com Castelo Branco (2007) uma forte tendência desse processo de urbanização foi o aumento do número de cidades médias. Ainda segundo a autora cidades médias se constituem nós da rede urbana, pois exercem um papel estratégico no desenvolvimento regional. 
O termo cidades médias, apesar de bastante difundido, não possui uma definição prescrita, pois não existe um padrão de cidade média, para identificá-las é necessário considerar um conjunto de variáveis para determinar se uma cidade é de médio porte ou não, tais como, tamanho demográfico, funções, dinâmicas intra-urbana, qualidade de vida, infra-estrutura, relações externas entre outras (SOARES, 2007).

Rocha (2005) ao discutir o processo de expansão urbana da cidade de Mossoró aponta que os caminhos da expansão urbana da referida cidade ocorreram em virtude da dinâmica econômica gerada pelas atividades salineiras, petrolífera e da fruticultura irrigada, entendendo-as como dirigentes da economia e da organização espacial da cidade:

O processo de expansão espacial e de modificações estruturais atinge áreas urbanas, suburbanas e, até mesmo, áreas consideradas rurais. Esses limites são dinâmicos, não só no espaço, mas também no tempo e obedecem aos interesses econômicos, sociais e políticos (ROCHA, 2005, p. 53).

Segundo Jacobi (2006) a dinâmica da urbanização pela expansão de áreas periféricas produziu um ambiente urbano segregado e altamente degradado, com efeitos muito graves sobre a qualidade de vida de sua população. Nesse sentindo, o principal desafio enfrentado pelas cidades nos dias atuais é que a mesma crie condições necessárias para assegurar a qualidade de vida a sua população, de modo que não interfira negativamente no meio ambiente, atuando preventivamente para evitar o crescimento do nível de degradação, notadamente nas regiões habitadas pelos setores mais carentes.

Estima-se que no Brasil exista mais de 11 milhões de pessoas vivendo em aglomerados subnormais, uma generalização dada pelo instituto para os assentamentos irregulares existentes no país, conhecidos como: favela, invasão, grota, baixada, comunidade, vila, entre outros (IBGE, 2010).

Conforme Silva e Travassos (2008) são características inerentes a essas áreas a existência de terras livres, a ausência de infra-estrutura instalada, a precariedade de serviços públicos, a longa distância dos centros urbanos, a baixa qualidade dos loteamentos e a clandestinidade generalizada. As autoras expõem que diante de tais características essas áreas têm o seu valor rebaixado no mercado imobiliário, o que possibilita o acesso por parte da população de baixa renda. Diante desta situação, a ausência do poder público na provisão de políticas públicas habitacionais de interesse social, fez com que o modelo de expansão periférica predominasse no contexto urbano brasileiro desde a década de 1950, no qual se transformou em uma das formas de acesso habitacional mais significativa do país.

Na década de 80, as cidades brasileiras sofreram um aumento significativo nos índices de criminalidade. Alguns estudos sociológicos apontam que o aumento das taxas de criminalidade nas cidades está associado ao acelerado crescimento urbano, que diante do rápido processo migratório levou a concentração de amplas massas isoladas nas periferias dos centros urbanos, sob condições precárias de pobreza e desorganização social (SILVA, 2002).

Com processo de industrialização, as cidades passaram a ser cada vez mais urbanas, o que afetou diretamente o modelo espacial da cidade, seu ambiente e as relações nelas estabelecidas. Gerando assim um ambiente hostil, permeado de relações fragilizadas. Embora a criminalidade 
não seja uma manifestação exclusivamente urbana, é nas cidades onde ela acontece com mais intensidade, aumentando a sensação de insegurança por parte da população (CERQUEIRA, 2012).

O espaço urbano é reflexo da organização e a dinâmica de cada sociedade. No Brasil, as áreas urbanas são marcadas por grandes desigualdades de renda, o que provoca heterogeneidades sócio-espaciais, ou seja, desigualdades socioeconômicas que fragmentam o espaço urbano em inúmeros territórios com atributos próprios e excludentes, que incidem na criminalidade e no enfraquecimento da cidadania (ROCHA; SILVA, 2005).

A espacialização das ocorrências de crimes em meio urbano tem sido cada vez mais expressiva, notadamente, por estudos que associam o desenho urbano a áreas com maior ou menor incidência de crimes. Entendendo, que o desenho pode contribuir para problema da criminalidade urbana, pela falta de apoio social. Silva (2002) aponta que em locais da cidade que apresentam um grande número de pessoas em condições pouco organizadas são mais propensos ao surgimento dos chamados bolsões de violência.

Desta forma, fatores relacionados com os aspectos urbanísticos e ambientais podem ser a explicação para a concentração de crimes em uma dada área. Os autores destacam que nos últimos anos tem sido cada vez mais explorado o estudo sobre os fatores ambientais em locais de grande ocorrência de crimes. A ideia central desses estudos é que a desordem e a degradação física do ambiente exercem influência direta sobre a ocorrência dos crimes (BEATO; ASSUNÇÃO, 2008).

\section{METOdOLOGIA}

Para a espacialização da criminalidade urbana do município de Mossoró utilizou-se dados secundários e quantitativos, como também pesquisa bibliográfica e documental para embasamento das análises. É necessário ressaltar que o ponto tratado sobre criminalidade se restringiu, a análise dos dados sobre homicídios, pois este representa o ápice da escala de violência, também pelo fato de os dados de homicídios serem mais confiáveis do que outros, tendo em vista que outros tipos de criminalidade muitas vezes não são registrados corretamente (ANDRADE; FIGUEREDO, 2005).

Para elaboração do mapa da criminalidade coletou-se dados sobre a ocorrência de homicídios no município de Mossoró, detalhado por bairros, entre o período de 2010 a 2013 no Instituto Técnico-Científico de Polícia. Salienta-se que a localização registrada pelo Instituto se refere à localização onde a vítima foi encontrada. Também foi utilizado o Sistema de Informações Geográficas software ArcGIS 10.1 e base cartográfica do Instituto Brasileiro de Geografia e Estatística.

Além disso, analisou-se dados de homicídios do Ministério da Saúde, através do Departamento de Informática do Sistema Único de Saúde (DATASUS) - Estatísticas Vitais Mortalidade dos anos de 1996 a 2011, disponibilizados virtualmente por meio do site (http://www2.datasus.gov.br); e dados das publicações do Mapas da Violência de 2002 a 2011 (WAISELFISZ, 2007, 2010, 2013), para subsidiar a compreensão da evolução histórica do número de homicídios no estado do Rio Grande do Norte e no município de Mossoró. 


\section{RESULTADOS E DISCUSSÕES}

A cidade de Mossoró vem passando nas últimas décadas por um momento de dinamismo nos processos de ocupação do espaço urbano, no qual pode ser verificado por meio da observância do rápido crescimento (SALLES, 2013). Além disso, município passou por transformações significativas na sua estrutura populacional, seguindo as tendências nacionais de transição acelerada da dinâmica demográfica (PESSOA, 2011). A Figura 1 apresenta o crescimento populacional do município de Mossoró em comparação ao estado.

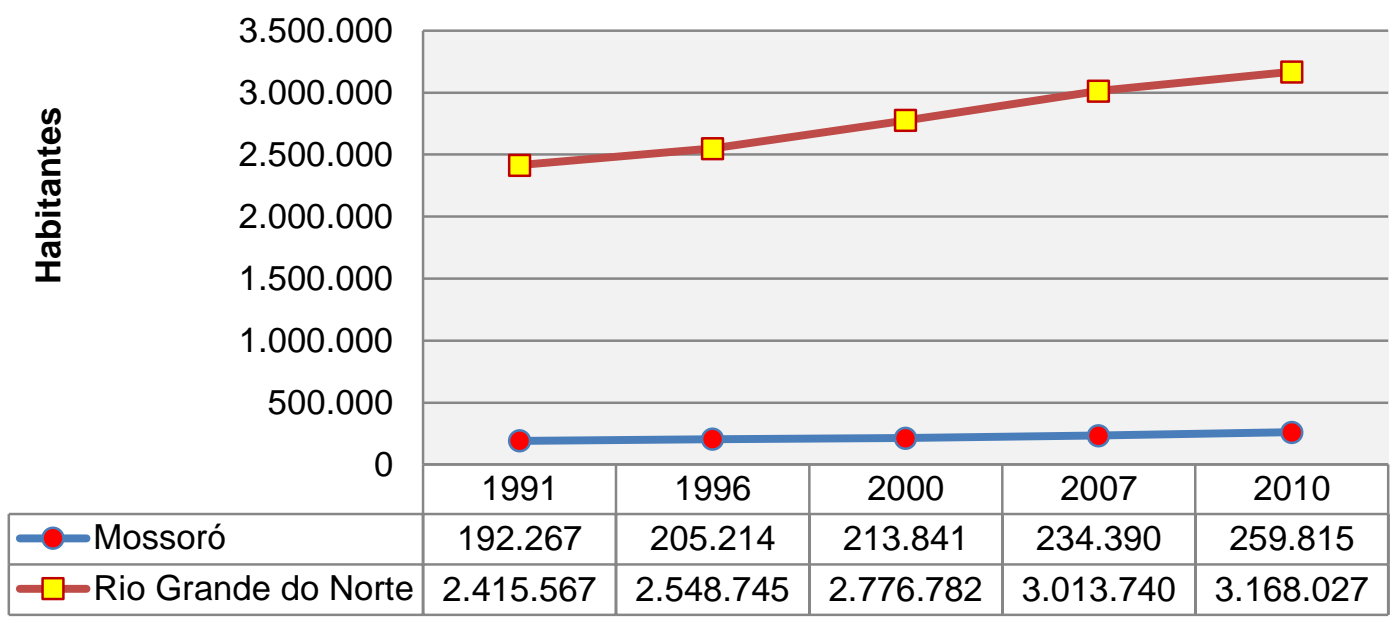

Figura 1: Crescimento demográfico do estado do Rio Grande do Norte e do município de Mossoró, 1992 a 2010.

Fonte: Adaptado do IBGE, 2010.

Analisando a série histórica de homicídios ocorridos no Estado do Rio Grande do Norte (RN) e no município de Mossoró por meio dos dados do DATASUS (FIGURA 2) e Mapas da Violência (FIGURA 3), observa-se que as taxas de homicídio têm apresentado um crescimento.

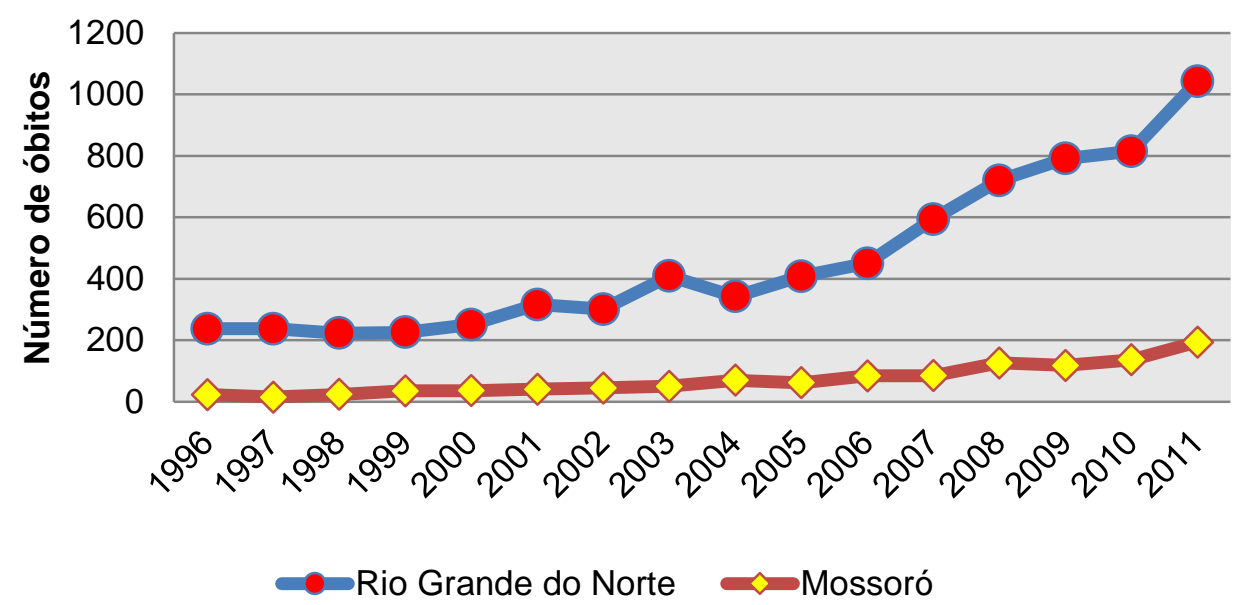

Figura 2: Evolução dos óbitos no Rio Grande do Norte e no município de Mossoró entre os anos de 1996 a 2011. Fonte: Pimenta (2014) com base nos dados do DATASUS.

Na Figura 3 são apresentados os números de óbitos por homicídio no estado do RN, na capital e no município de Mossoró. Observa-se que o número de homicídios registrados triplicou no estado do RN e na capital, enquanto no município de Mossoró esse número quadruplicou entre 
os anos de 2002 a 2011. Também podemos perceber que o município de Mossoró no ano de 2004, o número de óbitos praticamente igualou-se com a capital do estado, Natal.

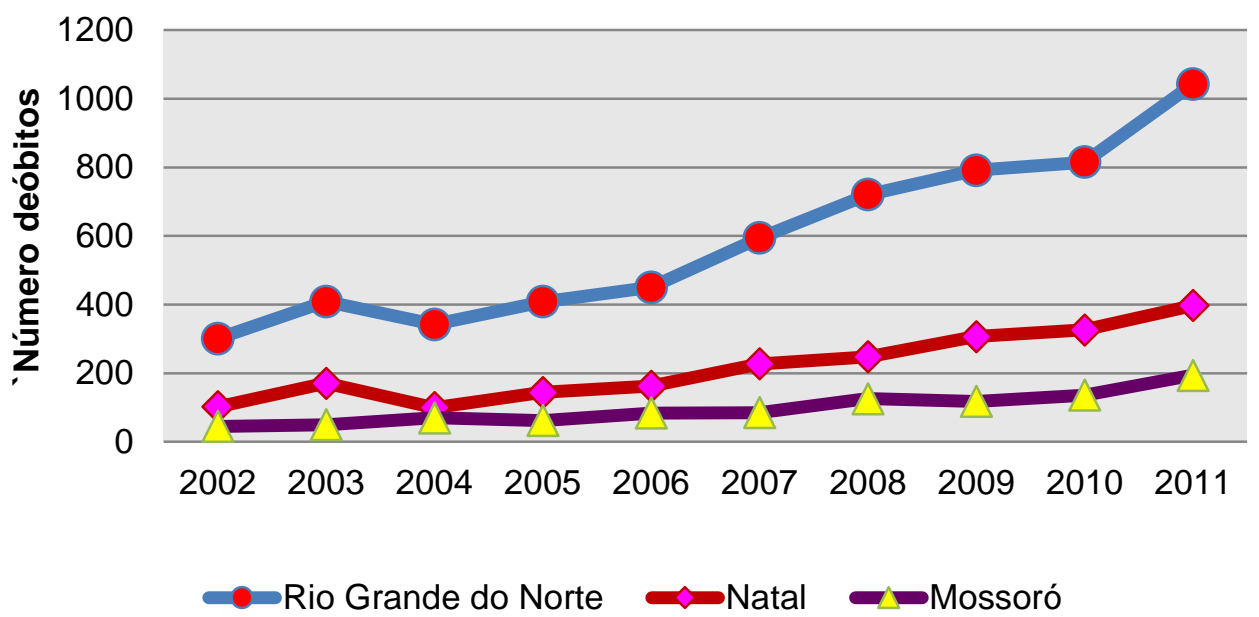

Figura 3: Óbitos por homicídio no Rio Grande do Norte, e nos municípios de Natal e Mossoró, 2002-2011. Fonte: Silva (2014) com base nos Mapas da Violência.

Durante o período de 2010 a 2013 foram registrados no município de Mossoró pelo ITEP 618 homicídios, dos quais 547 ocorreram na zona urbana, 55 na zona rural e 16 casos não foram identificados à localização do homicídio (FIGURA 4).

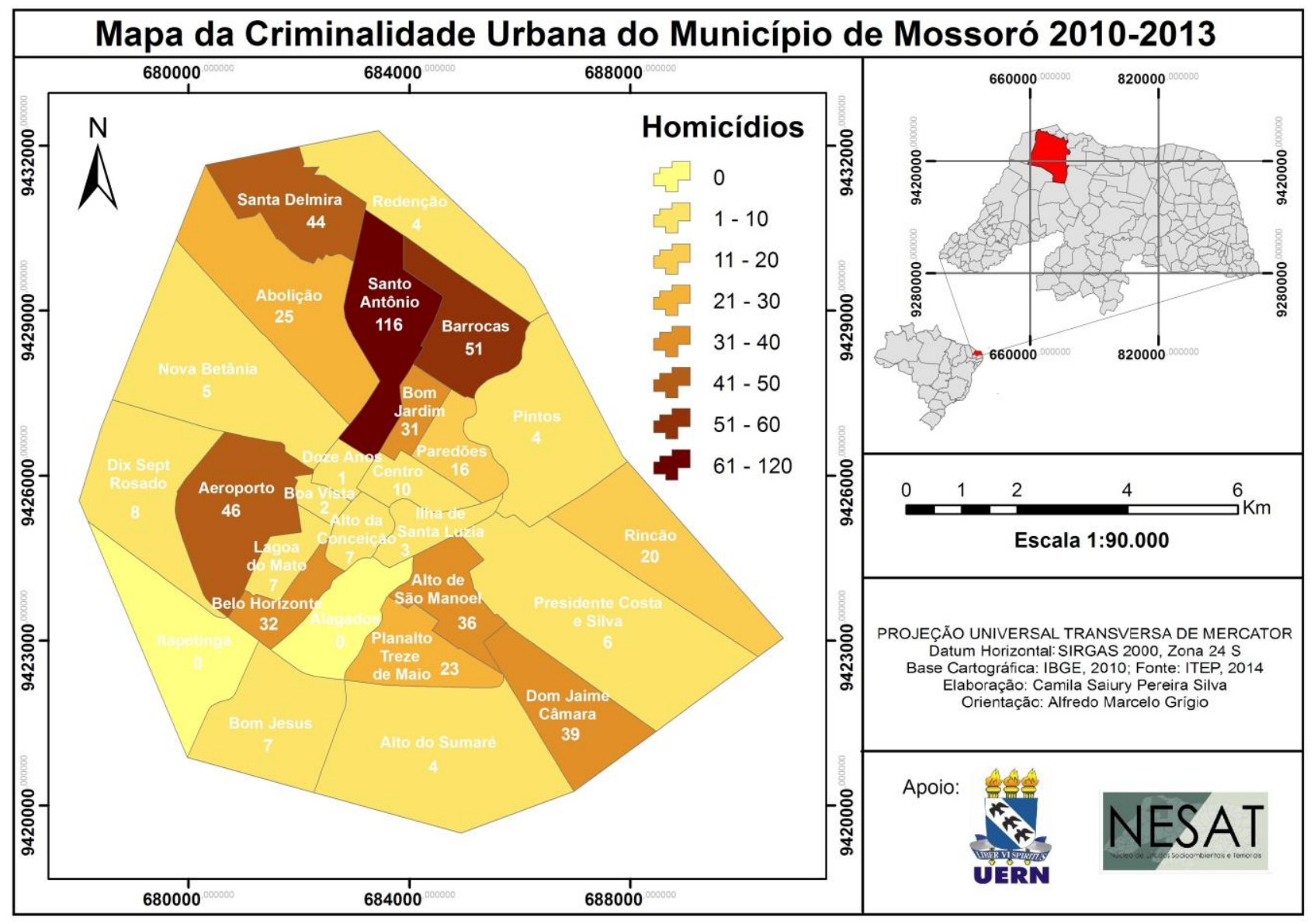

Figura 4: Mapa da criminalidade urbana do município de Mossoró, 2010-2013.

Fonte: Dados do ITEP, 2014. 
Verificou-se que o bairro Santo Antônio apresentou a maior incidência de homicídios em todos os anos avaliados. Observa-se deste modo que $21,2 \%$ dos homicídios ocorreram em apenas 4,9\% do perímetro urbano. Esse resultado corrobora com as pesquisas apontadas por Beato (2008) no qual identifica que fatores relacionados à ecologia urbana influenciam diretamente na distribuição espacial de determinados tipos de crimes, ou seja, existe uma concentração do número de crimes em um dado espaço geográfico.

O bairro Barrocas, segundo bairro de maior incidência com 9,5\% dos homicídios, não chega a representar a metade dos óbitos registrados no bairro Santo Antônio. Em seguida os bairros Aeroporto com 8,4\%, Santa Delmira com 7,9\% e Dom Jaime Câmara com 7,3\% dos homicídios.

Analisando a distribuição da criminalidade na zona urbana percebe-se que existe uma concentração dos homicídios em cinco bairros da cidade. Esses bairros possuem favelas em suas áreas, sendo elas: favela do Wilson Rosado no bairro Santo Antônio, Santa Helena no bairro Barrocas, favela do Ouro Negro no bairro Aeroporto, favela do Fio no bairro Santa Delmira e favela do Tranquilim no bairro Dom Jaime Câmara. Essas favelas são caracterizadas pela falta de equipamentos comunitários e serviços públicos essenciais (SALLES, 2013).

Dias (2013) ao espacializar a vulnerabilidade social de Mossoró destaca que as áreas periféricas dos bairros Santo Antônio, Barrocas e Santa Delmira apresentam vulnerabilidade social média alta a muito alta, ressaltando assim a necessidade de uma maior atenção por parte do poder público no que concernem as políticas públicas de cunho social.

Além disso, Andrade e Figueiredo (2005) apontam que própria criminalidade já constitui em si um indicador de vulnerabilidade, seja de risco social - inclusive de perder a própria vida -, seja dos custos econômicos dela decorrentes, assim como da perda da qualidade de vida derivada do medo e de outras inseguranças.

\section{CONCLUSÃO}

Os produtos cartográficos gerados ofereceram um olhar diferenciado sobre a criminalidade, indo além daquele fornecido pelos dados tabulares. Sabendo onde existe maior probabilidade de um crime ocorrer é possível traçar estratégias preventivas e políticas públicas de combate e controle da criminalidade e para uma gestão mais efetiva.

Nos anos analisados, percebe-se que os números de homicídios aumentaram progressivamente e apresentam uma forte tendência em se manter no mesmo ritmo. Essa tendência de crescimento pode ser verificada tanto pelos dados do ITEP, quanto pelos dados do DATASUS e do Mapa da Violência.

O presente artigo deve ser visto como uma primeira investigação sobre a questão da criminalidade na cidade de Mossoró. Portanto, espera-se que os resultados obtidos neste trabalho possam servir de subsídios para que os gestores públicos possam planejar a cidade levando em consideração à questão da criminalidade, principalmente vista sob os aspectos de natureza socioeconômica e urbanística, com vistas à melhoria da qualidade de vida de suas populações. Como também agregar elementos para outras pesquisas que visem estudar o planejamento urbano, de forma integrada, constituídas de todos os elementos que fazem parte da cidade. 


\section{REFERÊNCIAS BIBLIOGRÁFICAS}

1. ANDRADE, L. T. de; FIGUEIREDO, F. O. V. de. Vulnerabilidade social e criminalidade na Região Metropolitana de Belo Horizonte. Observatório das Metrópoles, 2005.

2. BEATO, C. (Org.). Compreendendo e avaliando: projetos de segurança pública. Belo Horizonte: Editora UFMG, 2008. $219 \mathrm{p}$

3. BEATO, C.; ASSUNÇÃO, R. Introdução ao uso de mapas para análise criminal. In: BEATO, C. (Org.). Compreendendo e avaliando: projetos de segurança pública. Belo Horizonte: Editora UFMG, 2008.p. $12-59$.

4. CASTELO BRANCO, M. L. G. Algumas Considerações sobre a Identificação de Cidades Médias. In: SPOSITO, Maria Encarnação Beltrão (Org.). Cidades Médias: espaços em transição. São Paulo: Expressão Popular, 2007. p. $89-111$.

5. CERQUEIRA, Y. M. S. F. Criminalidade, Sensação de Insegurança e "Desvinculação" do Lugar. III Seminário Internacional Urbicentros, Salvador, 2012.

6. DIAS, H. G. Identificação da vulnerabilidade socioambiental na área urbana de Mossoró-RN, a partir do uso de técnicas de análises espaciais. Dissertação (Mestrado em Ciências Naturais) Programa de Pós-Graduação em Ciências Naturais, da Faculdade de Ciências Exatas e Naturais da Universidade do Estado do Rio Grande do Norte. Mossoró, 2013.

7. IBGE. Instituto Brasileiro de Geografia e Estatística. Censo Demográfico 2010: Aglomerados subnormais. Rio de Janeiro, p.1-259, 2010.

8. _ Censo Demográfico 2010: Características da população e dos domicílios. Rio de Janeiro, 2011. Disponível em:<http://biblioteca.ibge.gov.br>. Acesso em: 29 de set. 2013.

9. JACOBI, P. Cidade e meio ambiente: percepções e práticas em São Paulo. 2a ed. São Paulo: Annablume, 2006.

10. MENEGAT, R; ALMEIDA, G. Sustentabilidade, Democracia e Gestão Ambiental Urbana. In: MENEGAT, R; ALMEIDA, G; SATTERTHWAITE, D (Org.). Desenvolvimento sustentável e gestão ambiental nas cidades: estratégias a partir de Porto Alegre. Porto Alegre: UFRGS, 2004.

11. PESSOA, Z. S. Aspectos Demográficos. In: PESSOA, Z. S. et al. (Org.). Como anda Mossoró: análise da conjuntura sócio-urbana, ambiental e político-institucional. Natal: UFRN, 2011.

12. PIMENTA, M. R. C. A política pública de segurança em Mossoró-RN: uma avaliação de processo da implantação da guarda civil (2009-2013). 2014. Dissertação. (Mestrado em Ciências Sociais) - Programa de Pós-Graduação em Ciências Sociais, da Universidade Federal do Rio Grande do Norte. Natal, 2014. 131 p.

13. PUGLISI, V. P. Meio Ambiente Urbano: Desenvolvimento Sustentável e Qualidade de Vida. 2006. Dissertação (Mestrado em Direito das Relações Sociais) - Universidade Católica de São Paulo, São Paulo, 2006.

14. ROCHA, A. P. B. Expansão urbana de Mossoró (período de 1980 a 2004): geografia dinâmica e reestruturação do território. Natal, RN: EDUFRN Ed. Da UFRN, 2005. p. 292.

15. ROCHA, C. P. A; SILVA, L. F. M; Paisagem do medo: um estudo do bairro da Pituba - Salvador BA. In: TERRA, C. G.; ANDRADE, R. (Org.). Paisagens Culturais: Interfaces entre Tempo e espaço na construção da Paisagem Sul-Americana. Rio de Janeiro: EBA, 2008. 
16. SALLES, M. C. T. Análise das Potencialidades e Fragilidades da Política Urbana e Ambiental do Município De Mossoró (RN). Dissertação (Mestrado em Ciências Naturais) - Programa de PósGraduação em Ciências Naturais, da Faculdade de Ciências Exatas e Naturais da Universidade do Estado do Rio Grande do Norte. Mossoró, 2013.

17. SANTOS, M. A urbanização brasileira. São Paulo. Editora da Universidade de São Paulo. $5^{\circ}$ ed. 2009.

18. SILVA, C. S. P. Levantamento e espacialização da criminalidade urbana do município de Mossoró-RN. 2014. Monografia. (Graduação em Gestão Ambiental) - Faculdade de Ciências Econômicas, da Universidade Federal do Rio Grande do Norte. Mossoró, 2014. 73 p.

19. SILVA, B. F. A. Criminalidade urbana violenta: uma análise espaço-temporal dos homicídios em Belo Horizonte. Fafich, UFMG, 2002.

20. SILVA, L. S; TRAVASSOS, L. Problemas ambientais urbanos: desafios para a elaboração de políticas públicas integradas. Cadernos metrópole, 2008. p. 27-47.

21. SOARES, B. R. Pequenas e Médias Cidades: Um estudo sobre as relações socioespaciais nas áreas de cerrado em Minas Gerais. In: SPOSITO, Maria Encarnação Beltrão (Org.). Cidades Médias: espaços em transição. São Paulo: Expressão Popular, 2007. p. 89 - 111.

22. WAISELFISZ, J. Mapa da Violência dos municípios brasileiros. Brasília: Organização dos Estados Ibero-Americanos para a Educação, a Ciência e a Cultura, 2007.

23. … Mapa da Violência: anatomia dos homicídios no Brasil. São Paulo: Instituto Sangari, 2010.

24. _. Mapa da Violência 2013: Homicídios e Juventude no Brasil. Rio de Janeiro: Centro Brasileiro de Estudos Latino-Americanos, 2013. 